\title{
Next Generation Science Standards
}

\section{National Nanotechnology Infrastructure Network (NNIN) Lessons}

Lesson: Bigger is Not Always Better

\section{Standards:}

HS-PS1-5. Apply scientific principles and evidence to provide an explanation about the effects of changing the temperature or concentration of the reacting particles on the rate at which a reaction occurs. 